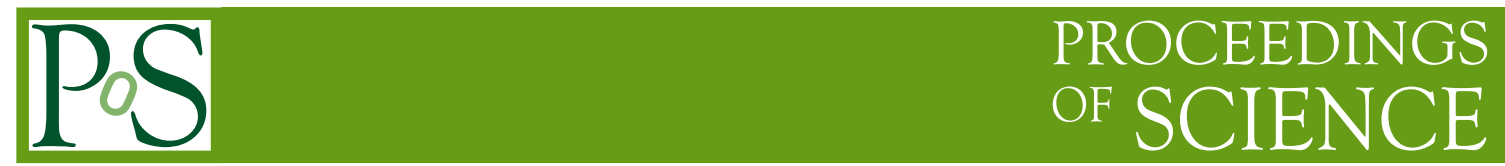

\title{
Trigger Issues for New Physics Searches in the CMS Experiment
}

\author{
Massimiliano Chiorboli on behalf of the CMS Collaboration \\ CERN, Università di Catania, INFN \\ E-mail: massimiliano.chiorboli@cern.ch
}

The CMS trigger system has been designed to cope with unprecedented luminosities and accelerator bunch-crossing rates of up to $40 \mathrm{MHz}$ at the LHC. We discuss the performance of the CMS trigger and some of the issues relevant to searches for new physics.

35th International Conference of High Energy Physics - ICHEP2010,

July 22-28, 2010

Paris France 
The CMS [1] trigger system is organized in two levels only: a hardware based L1 Trigger, based on coarse resolution objects reconstructed using the Calorimeter and the Muon System information, and a High Level Trigger (HLT). The HLT is based on 5000 CPU's which have access to the whole event information passed on by the L1. The first runs of the LHC machine have had a very low luminosity, lower than $10^{29} \mathrm{~cm}^{-2} \mathrm{~s}^{-1}$. The trigger tables designed for the start-up phase were very simple, based essentially on single and double objects (jets, electrons, muons, missing trasverse energy), with very low thresholds applied on their $\mathrm{p}_{T}$. The followed strategy was: simplicity to commission, debug and understand; inclusiveness, to be able to select also the unexpected; robustness against pathological events or against events having more hits than the ones foreseen with the Monte Carlo simulations; redundancy, so that a signal can be selected by more than one trigger, and is hence selected also in case one of the triggers has problems. The various triggers have been constantly monitored. An example can be found in fig. 1 in which the turn-on curves for electrons and jets are shown [2].

The luminosity has since gradually increased up to $2 \times 10^{32} \mathrm{~cm}^{-2} \mathrm{~s}^{-1}$ in October 2010 . When the luminosity increases, new conditions must be applied to the trigger definitions to keep the rates at acceptable levels. Prescales can be applied to the triggers with the lowest thresholds. Searches for new physics cannot rely on prescaled triggers, even if events useful for monitoring or calibration are usually collected by prescaled triggers. Single and double objects higher threshold triggers can be defined in order to keep the rate low without any prescale. For new physics searches, the simple single and double object triggers can cut away an entire range of spectrum of interesting physics if they have a too large threshold. Dedicated triggers have to be studied.

Supersymmetry events in collider experiments are characterized by final states containing jets, MET and possibly leptons. The CMS experiment defines the HT as the scalar sum of the jets passing a given threshold. A trigger based on it has been largely used for runs with luminosities up to $2 \times 10^{32} \mathrm{~cm}^{-2} \mathrm{~s}^{-1}$ as the main trigger for the hadronic supersymmetric analyses. Also some
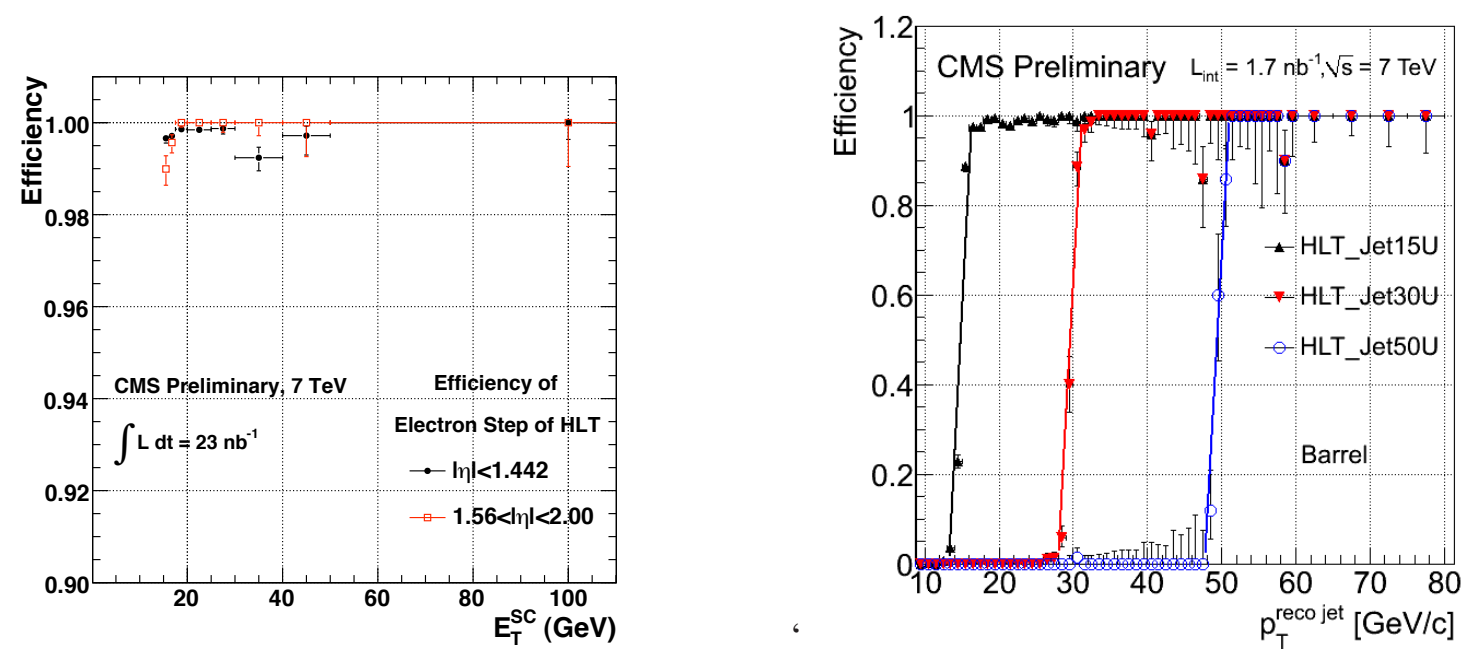

Figure 1: Left: the HLT_Ele15 efficiency for an offline reconstructed electron passing the HLT_Photon15 trigger as a function of the SuperCluster $E_{T}$. Right: the HLT_Jet15, HLT_Jet30 and HLT_Jet50 efficiencies for offline jets as a function of the jet $\mathrm{p}_{T}$ for jets in the barrel. 
leptonic analyses have exploited such a trigger: in many cases an analysis based on leptons in the final states has been performed using both an HT trigger or a trigger based on leptons. The usage of hadronic triggers for analyses with leptons in the final states gives the possibility to request leptons with a very low offline threshold.

The 2011-2012 LHC run is expected to reach much higher luminosities than the one reached in 2010: a luminosity of $5 \times 10^{33} \mathrm{~cm}^{-2} \mathrm{~s}^{-1}$ is foreseen in an optimistic scenario. Triggers for Supersymmetry are being redesigned to cope with such a large luminosity. For hadronic analysis, HT cannot be considered anymore the main trigger, due to the very high threshold necessary to have a sustainable rate. Other solutions will be used, like the combination in a cross trigger of HT and MHT (defined as the opposite of the vectorial sum of the $\mathrm{p}_{T}$ of all the jets in the event passing a given threshold) or the Effective Mass trigger, where $M_{e f f}=\mathrm{HT}+\mathrm{MHT}$. For leptonic analyses, the combination of leptons and HT in a cross trigger is the chosen strategy. For analyses with two leptons in the final state, pure dilepton triggers can also be used: in case of muons they are expected to have a sustainable rate even at high luminosities; for electrons, the rate can be kept under control by applying some identification cuts. For trilepton analyses, dedicated trilepton triggers can be implemented, with limited rate, exploiting the same L1 seeds used by the dilepton triggers.

For other searches beyond the Standard Model, other trigger issues have to be faced. Hidden Valley models predict displaced verteces. An outside-in muon trigger algorithm has been developed to avoid a track seeding based on the pixel detector information, which can cut signals for muons produced far from the interaction point. Heavy Stable Charged Particles (HSCP) behave in some cases as heavy muons. Muon triggers can be used in such cases, but only for particles having $\beta>0.6$. For slower particles, a wrong bunch crossing assigment can occur because of the time of flight being greater than $25 \mathrm{~ns}$. Other triggers, like a missing transverse energy based trigger, have to be used in these cases, even if they are based on the rest of the event and not on the long lived particle itself and is hence model dependent. In some HSCP models, the decay time is so long that the particles can be trapped in the detector and decay some time later (from $\mu$ s to months depending on the model). The CMS Collaboration has developed a dedicated trigger [3] for this peculiar scenario, based on a calorimeter trigger looking at particles trapped in the CMS HCAL during periods of no proton-proton or heavy-ion collisions. The trigger can be operated in both the interfill period, when the beam is off, or in the beamgap, exploiting a beam position monitor to request the absence of the beam. The discovery is possible from lifetimes of the order of $100 \mathrm{~ns}$ to months.

\section{References}

[1] The CMS Collaboration, JINST 3:S08004,2008.

[2] The CMS Collaboration, CMS-DP-2010-032.

[3] The CMS Collaboration, CMS-PAS-EXO-10-003. 